\title{
Threat assessment for driver assistance systems using interval-based techniques
}

\author{
Esteban R. Gelso* Mohammad Ali ${ }^{*, * *}$ Jonas Sjöberg* \\ * Chalmers University of Technology, Dept. of Signals and Systems, \\ 41296 Göteborg, Sweden (e-mail: \{gelso, jonas.sjoberg\}@chalmers.se). \\ ** Volvo Car Corporation, Active Safety $\& 3$ Chassis, 96410/PV4A, \\ 40531 Göteborg, Sweden (e-mail: mali21@volvocars.com)
}

\begin{abstract}
We present a threat assessment algorithm for driver assistance systems in which mathematical vehicle and driver models are used to explicitly account for the vehicle and driver behavior. For the application considered in this paper, requirements that the vehicle stays on the road while operating in a stable operating region are expressed as constraints on the vehicle states which need to be satisfied over a finite time horizon. The threat assessment problem is then formulated as a constraint satisfaction problem, solved through interval-based techniques considering bounded uncertainty in the measurements. Experimental results demonstrate the algorithms ability to detect unsafe operation in advance.
\end{abstract}

Keywords: Semi-Autonomous Vehicles, Active Safety, Constraint Satisfaction Problems, Bounded Uncertainty, Intervals.

\section{INTRODUCTION}

The introduction of electronic stability control systems in passenger vehicles has contributed significantly to the reduction of fatal and severe traffic accidents, specially those involving vehicles unintentionally leaving the road due to loss of control, Ferguson (2007). Unintended roadway departures however still account for the highest share of traffic related fatalities, Najm et al. (2003); LeBlanc et al. (2006), and are thus still a highly significant problem.

Currently, a wide range of driver assistance systems, which also reduce unintended roadway departures are being introduced in passenger cars. Curve speed warning systems e.g. utilize sensor information about the oncoming road and warn drivers if they are about to enter a curve too fast LeBlanc et al. (2006). By giving the driver the opportunity to reduce speed in advance, the curve speed warning system thus reduces the risk of losing control in the oncoming curve, Ali et al. (2009). Lane guidance systems are another type of driver assistance system which also contributes in reducing unintended roadway departures. Lane guidance systems warn or assist the driver through a steering intervention upon crossing a lane marking, Eidehall (2007), and can, in contrast to electronic stability control systems, be efficient in situations where the driver departs the road due to drowsiness or distraction.

A common problem in such safety oriented driver assistance systems is to determine in which situations drivers need assistance. On one hand, such systems need to detect critical situations and adequately assist the driver when necessary. On the other hand, alerts or interventions which drivers consider unnecessary, contribute negatively to drivers confidence in the systems and it is therefore crucial that false alerts are always avoided, Ali and Sjöberg (2009). In many of the lane guidance systems proposed in the literature this aspect is given little or no attention, while focus is instead on the vehicle control in case of an intervention. In this paper, the process of determining whether an intervention is required is referred to as threat assessment. A review of proposed lane guidance systems and threat assessment methods in the literature can be found in Ali (2010).

The focus of this manuscript is on finding reliable and computationally efficient threat assessment methods for driver assistance systems. In particular we start from the method presented in Falcone et al. (2010) where mathematical vehicle and driver models are used to explicitly account for the vehicle and driver behavior. The requirements that the vehicle stays on the road while operating in a stable operating region are expressed as constraints on the vehicle states which need to be satisfied over a finite time horizon. The threat assessment problem is then formulated as a constraint satisfaction problem and solved through reachability analysis for linear systems.

We present a preliminary study, evaluating the potential of the approximative interval-based consistency techniques in solving this problem. Interval-based consistency techniques have been implemented and applied in several different domains, such as model-based fault detection and diagnosis, robust control, and robotics, Gelso (2009); Jaulin et al. (2001). These techniques are particularly beneficial for handling uncertainties associated with e.g. modeling and measurement errors. In addition, the interval based methods can be used to obtain approximative solutions to nonconvex constraint satisfaction problems, enabling the possibility to extend the present method with nonlinear models and constraints. Compared to previous work, we account for uncertainties in the state measurement and show that we obtain reliable results, with low 


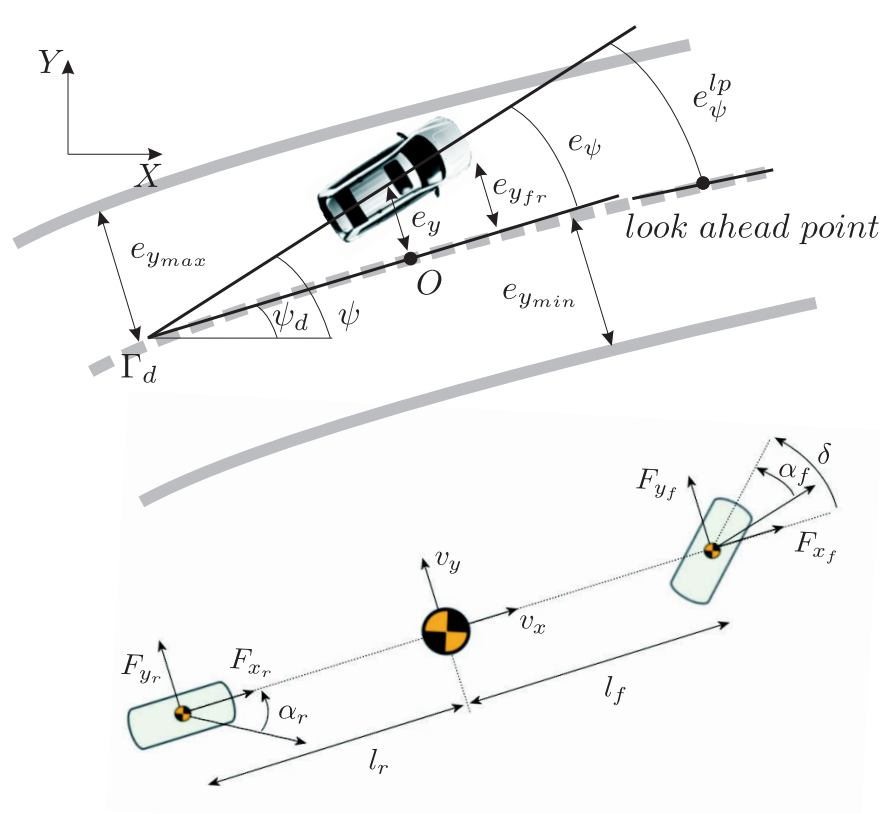

Fig. 1. Vehicle modeling notation.

computational burden. The method is validated using experimental data.

The rest of the paper is organized as follows. Section 2 presents the mathematical models used in the threat assessment algorithm to describe the vehicle and driver behavior. In Section 3, the threat assessment problem is stated as a constraint satisfaction problem. Section 4 introduces the fundamental concepts of interval analysis and the interval-based consistency techniques used to solve the constraint satisfaction problem. The proposed algorithm for the threat assessment problem is presented in Section 5. Section 6 analyzes the experimental results. Finally, Section 7 discusses the conclusions and some directions for future work.

\section{MATHEMATICAL MODELS}

In this section, we present the mathematical models used in the threat assessment algorithm to account for the vehicle and driver behavior.

\subsection{Vehicle model}

To describe the vehicle motion within the lane, we use a standard single-track vehicle model Rajamani (2006),

$$
\begin{aligned}
m \dot{v}_{y} & =-m v_{x} \dot{\psi}+2\left[F_{y_{f}}+F_{y_{r}}\right], \\
J_{z} \ddot{\psi} & =2\left[l_{f} F_{y_{f}}-l_{r} F_{y_{r}}\right], \\
\dot{e}_{\psi} & =\dot{\psi}-\dot{\psi}_{d}, \\
\dot{e}_{y} & =v_{y}+v_{x} e_{\psi},
\end{aligned}
$$

where, $m$ and $J_{z}$ denote the vehicle mass and yaw inertia, respectively, $F_{y_{f}}, F_{y_{r}}$ are the lateral tire forces at the front and rear axles, respectively, and the rest of the symbols are defined in Figure 1.

The lateral tire forces are calculated as,

$$
F_{y_{i}}=-C_{i} \alpha_{i}, \quad i \in\{f, r\}
$$

with $C_{i}$, as cornering stiffness coefficients at the two axles and $\alpha_{i}$, tire slip angles which, assuming small angles, are computed as,

$$
\begin{aligned}
\alpha_{f} & =\frac{v_{y}+l_{f} \dot{\psi}}{v_{x}}-\delta, \\
\alpha_{r} & =\frac{v_{y}-l_{r} \dot{\psi}}{v_{x}},
\end{aligned}
$$

where $\delta$ denotes the steering angle at the front wheel.

Remark 1. In general, the relation between lateral tire force and tire slip angle is nonlinear. In normal driving conditions however, slip angles are kept in a small region corresponding to stable driving. In this region, the linear model (2) provides a good approximation, Rajamani (2006).

Remark 2. We note that $\dot{\psi}_{d}$ in (1c) enters the system equations as a disturbance. In an online application the road curvature $c(s)$, where $s$ denotes distance along the road ahead of the vehicle, might be obtained from a digital map or through a vision system. Assuming $\dot{s} \approx v_{x}, \dot{\psi}_{d}$ can then be approximated through the relation $\dot{\psi}_{d}=c v_{x}$.

\subsection{Driver model}

The threat assessment method proposed in this paper utilizes a mathematical description of driver behavior. In the literature, there's a huge range of approaches to driver modeling, see e.g. Cacciabue (2007). In the proposed threat assessment algorithm we use a low complexity model. The steering angle $\delta$ is computed as,

$$
\begin{aligned}
\delta & =K_{y} e_{y}+K_{\psi} e_{\psi}^{l p}, \\
& =K_{y} e_{y}+K_{\psi} e_{\psi}+K_{\psi} \Delta \psi_{d},
\end{aligned}
$$

where $e_{\psi}^{l p}$ is an orientation error, with respect to the orientation of the road at time $t_{l p}$ ahead of the vehicle, as illustrated in Figure 1. $K_{y}, K_{\psi}$, and $t_{l p}$ are considered as parameters of the driver model.

Remark 3. The driver model parameters $K_{y}, K_{\psi}$, and $t_{l p}$ are in general both driver and situation dependant and might be estimated online in a vehicle application. Estimation results of these parameters are demonstrated in Falcone et al. (2010).

Remark 4. The disturbance $\Delta \psi_{d}$, describes the road geometry and might in an online application be obtained from data stored in a digital map. Alternatively, as noted in Remark 2, the road curvature $c(s)$, might be available through a vision system. In such case, assuming constant velocity, $\Delta \psi_{d}$, can be computed as,

$$
\Delta \psi_{d}=\int_{0}^{t_{l p}} c\left(v_{x} \tau\right) v_{x} d \tau .
$$

\subsection{Driver controlled vehicle model}

We combine the models (1)-(4) and write the combined vehicle and driver model in the following compact form,

$$
\dot{\mathbf{x}}(t)=A \mathbf{x}(t)+E \mathbf{w}(t),
$$


where $\mathbf{x}=\left[v_{y}, \dot{\psi}, e_{\psi}, e_{y}\right]^{T}$ is the state vector, $\mathbf{w}=$ $\left[\dot{\psi}_{d}, \Delta \psi_{d}\right]^{T}$ the disturbance vector, and the matrices $A$ and $E$ are omitted due to lack of space.

\subsection{System Constraints}

Next we express the requirements that the vehicle stays in the lane while operating in a stable operating region as constraints on the vehicle state.

Let $e_{y_{i j}}, i \in\{f, r\}, j \in\{l, r\}$, be the distances of the four vehicle corners from the lane centerline. $e_{y_{f r}}$ is shown in Figure 1. Assuming small orientation error, $e_{y_{i j}}$ can be written as

$$
\begin{array}{ll}
e_{y_{f l}}=e_{y}+\frac{c}{2}+a e_{\psi}, & e_{y_{f r}}=e_{y}-\frac{c}{2}+a e_{\psi}, \\
e_{y_{r l}}=e_{y}+\frac{c}{2}-b e_{\psi}, & e_{y_{r r}}=e_{y}-\frac{c}{2}-b e_{\psi},
\end{array}
$$

where $c$ is the vehicle width, $a$ and $b$ are the distances of the center of gravity from the front and rear vehicle bumpers, respectively.

The requirement that the vehicle stays in the lane is then expressed,

$$
-e_{y_{\max }} \leq e_{y_{i j}} \leq e_{y_{\max }}
$$

Further, the requirement that the vehicle operates in stable operating conditions is ensured by limiting the tire slip angles $\alpha_{i}, i \in\{f, r\}$ (see Remark 1),

$$
\alpha_{i_{\text {min }}} \leq \alpha_{i} \leq \alpha_{i_{\max }}
$$

We recall that the tyre slip angles $\alpha_{i}$ are affine functions of the state vector $\mathbf{x}$ (see equations (3)-(4)), hence the constraints (8)-(9) can be compactly written as,

$$
H_{t} \mathbf{x}(\mathbf{t}) \leq h_{t} .
$$

The constraints matrices $H_{t}, h_{t}$ are omitted due to lack of space.

Remark 5. We observe that the constraints matrices $H_{t}, h_{t}$ in (10) are situation dependant. Several of the parameters in $H_{t}, h_{t}$ like e.g. $e_{y_{\max }}$ and $\Delta \psi_{d}$ might vary over time.

\section{THREAT ASSESSMENT AS A CONSTRAINT SATISFACTION PROBLEM}

In this section we formulate the threat assessment problem as a Constraint Satisfaction Problem (CSP). At each time instant, if the vehicle state does not satisfy the constraints (10), the vehicle's operation can be considered unsafe. The threat assessment problem is therefore formulated as the problem of evaluating whether the evolution of the vehicle state over a future finite time horizon will satisfy a set of predefined constraints.

Denote by,

(1) $\mathcal{V}=\left\{z_{1}, \ldots, z_{n}\right\}$, a set of numeric variables

(2) $\mathcal{D}=\left\{\mathcal{Z}_{1}, \ldots, \mathcal{Z}_{n}\right\}$, a set of domains where $\mathcal{Z}_{i}$, a set of numeric values, is the domain associated with the variable $z_{i}$
(3) $\mathcal{C}(\mathbf{z})=\left\{C_{1}(\mathbf{z}), \ldots, C_{m}(\mathbf{z})\right\}$, a set of constraints where a constraint $C_{i}(\mathbf{z})$ is determined by a numeric relation (equation, inequality, inclusion, etc.) linking a set of variables under consideration.

We let $\mathcal{C S P}=(\mathcal{V}, \mathcal{D}, \mathcal{C}(\mathbf{z}))$, denote a CSP and introduce the following definition,

Definition 1. The solution of a $\mathrm{CSP}, \operatorname{solution}(\mathcal{C S P}=$ $(\mathcal{V}, \mathcal{D}, \mathcal{C}(\mathbf{z})))$ is the set of numerical variables $\Sigma$ for which all the constraints $C_{i} \in \mathcal{C}$ can be satisfied i.e.,

$$
\Sigma=\left\{\mathbf{z} \in \mathcal{Z} \mid C_{i}(\mathbf{z}) \text { holds } \forall C_{i} \in \mathcal{C}\right\}
$$

The threat assessment CSP is formulated in discrete time, the continuous time system (6) is therefore discretized with a sampling time $T_{s}$ to obtain the discrete time constrained system,

$$
\begin{aligned}
& \mathbf{x}(k+1)=A^{d} \mathbf{x}(k)+E^{d} \mathbf{w}(k) \\
& \text { subj. to } \\
& H_{k} \mathbf{x}(k) \leq h_{k} .
\end{aligned}
$$

Assuming measurements of the state vector at time step $k$ available, the threat assessment CSP over a 1-step horizon can now be stated as,

$$
\begin{aligned}
& \mathcal{V}=\{\mathbf{x}(k), \mathbf{x}(k+1), \mathbf{e}(k)\} \\
& \mathcal{D}=\left\{\mathcal{X}_{k}, \mathcal{X}_{k+1}, \mathcal{E}_{k}\right\} \\
& \mathcal{C}=\left\{H_{k+1} \mathbf{x}(k+1) \leq h_{k+1}\right. \\
& \mathbf{x}(k+1)=A^{d} \mathbf{x}(k)+E^{d} \mathbf{w}(k) \\
& H_{k} \mathbf{x}(k) \leq h_{k} \\
&\tilde{\mathbf{x}}(k)=\mathbf{x}(k)+\mathbf{e}(k)\} .
\end{aligned}
$$

Where $\tilde{\mathbf{x}}(k)$ is the measurement vector of the state variables, and $\mathbf{e}(k)$ represents the uncertainty associated with the measurements. For each measurement, the uncertainty is considered unknown but bounded, i.e. $\mathbf{e}(k) \in \mathcal{E}_{k}$.

The $N$-step threat assessment CSP can be formulated by repetition of (13).

\section{BACKGROUND ON INTERVAL TECHNIQUES}

Several methods can be used to find the solution $\Sigma$ to a CSP (Definition 1). This section gives a brief introduction to interval based consistency techniques, which have been used in the results presented in this paper. Section 4.1 provides basic definitions in interval mathematics, which are used in the interval techniques for CSPs in Section 4.2.

\subsection{Basic interval mathematics}

Interval mathematics is a generalization in which interval numbers replace real numbers and interval arithmetic replaces real arithmetic. The interval analysis, has been introduced in Moore (1966).

Definition 2. A real interval $[p]=[p, \bar{p}]=\{p \mid p \leq p \leq \bar{p}\}$ is a connected and closed subset of $\mathbb{R}$. Denote by $\mathbb{R}$ the set of all real intervals of $\mathbb{R}$.

Definition 3. An interval real vector [z], also called a box, is a subset of $\mathbb{R}^{n}$ and can be defined as the Cartesian product of $n$ intervals.

$$
[\mathbf{z}]=\left[z_{1}\right] \times\left[z_{2}\right] \times \ldots \times\left[z_{n}\right]
$$


Definition 4. If $\bullet$ denotes the classical operations $\{+,-$, $*, /\}$ on real numbers $p$ and $q$, i.e. addition, subtraction, multiplication and division, respectively, then the operations associated with interval numbers $[p]$ and $[q]$ are

$$
[p] \bullet[q]=\{p \bullet q \in \mathbb{R} \mid p \in[p], q \in[q]\}
$$

Definition 5. The intersection of two intervals is defined by

$$
[p] \cap[q]=\{r \in \mathbb{R} \mid r \in[p], r \in[q]\}
$$

Definition 6. The union of two intervals is defined by

$$
[p] \cup[q]=\{r \in \mathbb{R} \mid r \in[p] \text { or } r \in[q]\}
$$

Definition 7. The interval hull of the union of two intervals $[p] \cup[q]$, is denoted by $[p] \sqcup[q]$, and is the smallest interval such that $[p] \cup[q] \subseteq[p] \sqcup[q]$.

Definition 8. Let $\mathbf{f}: \mathbb{R}^{n} \rightarrow \mathbb{R}^{m}$. Denote by, $\mathbf{f}([\mathbf{z}])=$ $\{\mathbf{f}(\mathbf{z}) \mid \mathbf{z} \in[\mathbf{z}]\}$, the range of $\mathbf{f}$ over an interval vector $[\mathbf{z}]$. Then an interval function $[\mathbf{f}]: \mathbb{\mathbb { R } ^ { n }} \rightarrow \mathbb{I}^{m}$, is an inclusion function for $\mathbf{f}$ if,

$$
\forall[\mathbf{z}] \in \mathbb{I} \mathbb{R}^{n}, \mathbf{f}([\mathbf{z}]) \subseteq[\mathbf{f}]([\mathbf{z}]) .
$$

\subsection{Interval techniques for constraint satisfaction problems}

In interval-based consistency techniques, the solution to a CSP, $\Sigma$, is approximated by one or several intervals or boxes $[\mathbf{z}]_{i}$ (Definitions 2 and 3 ). The solution, $\Sigma$, is obtained by pruning the initial domain of the variables of the CSP, through successive elimination of subboxes which cannot contain the solution. The consistency techniques most commonly used are known as Hull-consistency (also called 2B-consistency) and Box-consistency, or are variations of them, Benhamou et al. (1999).

Hull-consistency algorithms decompose the original constraints into a set of primitive constraints, the constraints are then enforced by interval arithmetic operations (Definitions 4-8). In general, wrapping of generic sets into boxes, decomposition of constraints and use of interval operations lead to overestimation of the solution $\Sigma$. In this manuscript we utilize interval techniques which provide an outer approximation $\hat{\Sigma}$ that is guaranteed to enclose the true solution $\Sigma$. The choice of such techniques is commented in Section 5. For a rigorous treatment of consistency techniques for CSPs we refer the reader to Collavizza et al. (1999); Jaulin et al. (2001).

\section{INTERVAL-BASED THREAT ASSESSMENT ALGORITHM}

Denote by $\mathbf{W}_{k}=\left[\mathbf{w}_{k}, \mathbf{w}_{k+1}, \ldots, \mathbf{w}_{k+N-1}\right]$ a sequence of disturbance samples over the horizon $[k, k+N-1]$. We formulate an $N$-step threat assessment CSP and enforce the constraints (12b), to hold for the system (12a), at each time step over a finite time horizon of $N$ steps. An interval-based branch and prune algorithm is then used to solve the threat assessment CSP. If the obtained solution $\hat{\Sigma}$ is empty, a violation of the constraints (10) can be expected within the horizon of $N$-steps and a flag notSafe is set, activating an autonomous intervention or warning. The threat assessment algorithm is outlined in Algorithm 1.

Remark 6. The initiation of an autonomous intervention in a situation where the driver is not in need of assistance might be both dangerous and intrusive. It is therefore

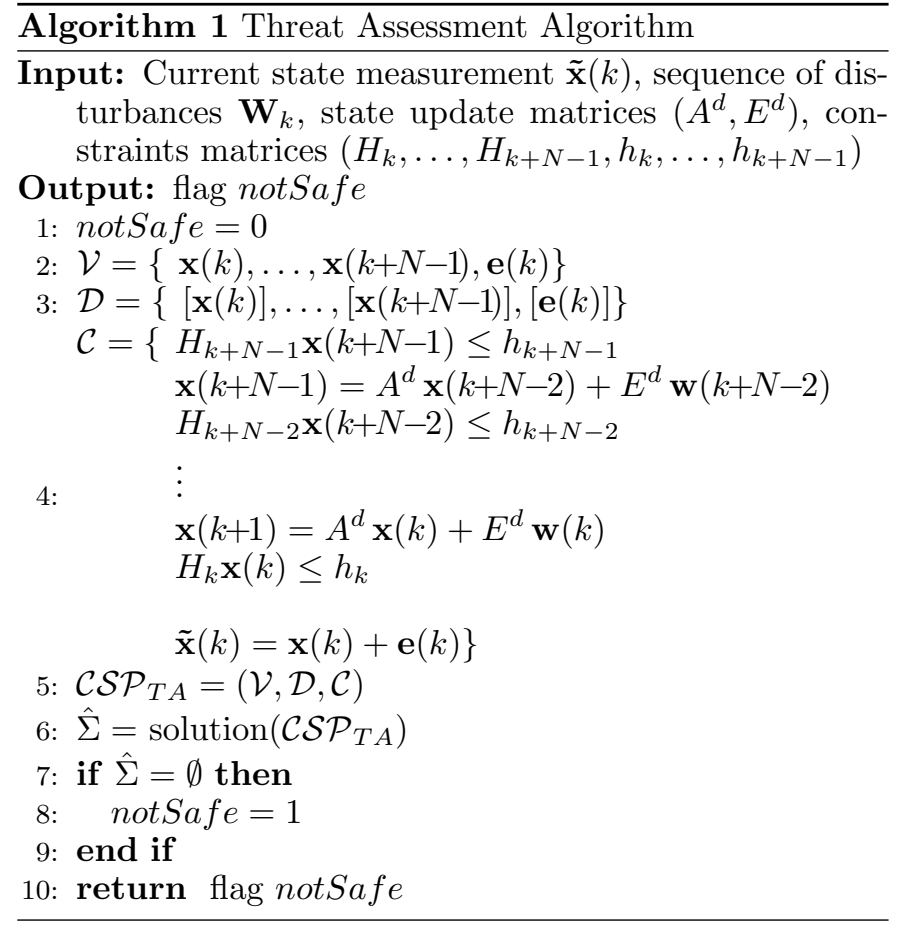

important that such an intervention is issued only once it can be guaranteed that the driver is in need of assistance. Since the interval based solver guarantees that $\Sigma \subseteq \hat{\Sigma}$, $\hat{\Sigma}=\emptyset \Longrightarrow \Sigma=\emptyset$, hence, based on the model (12), a violation of the constraints (10) can be guaranteed within the horizon of $N$-steps.

\section{EXPERIMENTAL RESULTS}

Algorithm 1 has been validated off-line against experimental data acquired on a test track. Measurements of the state variables and disturbances were collected using a differential GPS unit, a built-in high precision inertial measurement unit and a digital map.

The vehicle and driver parameters used in the algorithm are shown in Table 1, and the parameters of the constraints on the vehicle position and slip angles are stated in Table 2. Sampling time for measurements is taken as $T_{s}=0.01 \mathrm{~s}$, and the prediction horizon $N=35$ steps. As noted in Remark 3, the driver model parameters might be estimated recursively and updated online. In this paper however, the driver model parameters shown in Table 1 have been estimated offline on a batch of measurement data.

Table 1. Vehicle and driver parameters

\begin{tabular}{|l|l|l|l|}
\hline$m[\mathrm{~kg}]$ & $J_{z}\left[\mathrm{kgm}^{2}\right]$ & $l_{f}[\mathrm{~m}]$ & $l_{r}[\mathrm{~m}]$ \\
\hline 1695 & 2617 & 1.14 & 1.50 \\
\hline \hline$a[\mathrm{~m}]$ & $b[\mathrm{~m}]$ & $c[\mathrm{~m}]$ & $C_{f}[\mathrm{kNm} / \mathrm{rad}]$ \\
\hline 1.83 & 2.69 & 1.77 & 54 \\
\hline \hline$t_{l p}[\mathrm{~s}]$ & $K_{\psi}[-]$ & $K_{y}\left[\mathrm{~m}^{-1}\right]$ & $C_{r}[\mathrm{kNm} / \mathrm{rad}]$ \\
\hline 0.82 & -0.005 & -0.002 & 45 \\
\hline
\end{tabular}

Consider the situation shown in Figure 2, where the vehicle enters a curve with a speed of approximately $84 \mathrm{~km} / \mathrm{h}$. We observe that for such a narrow curve the vehicle speed in this situation is too large. As seen in Figure 2, the 
Table 2. Parameters of the constraints on the vehicle state

\begin{tabular}{|l|l|l|}
\hline$e_{y_{\max }}[\mathrm{m}]$ & $\alpha_{f_{\min }}, \alpha_{r_{\min }}\left[^{\circ}\right]$ & $\alpha_{f_{\max }}, \alpha_{r_{\max }}\left[^{\circ}\right]$ \\
\hline 1.56 & -4 & 4 \\
\hline
\end{tabular}

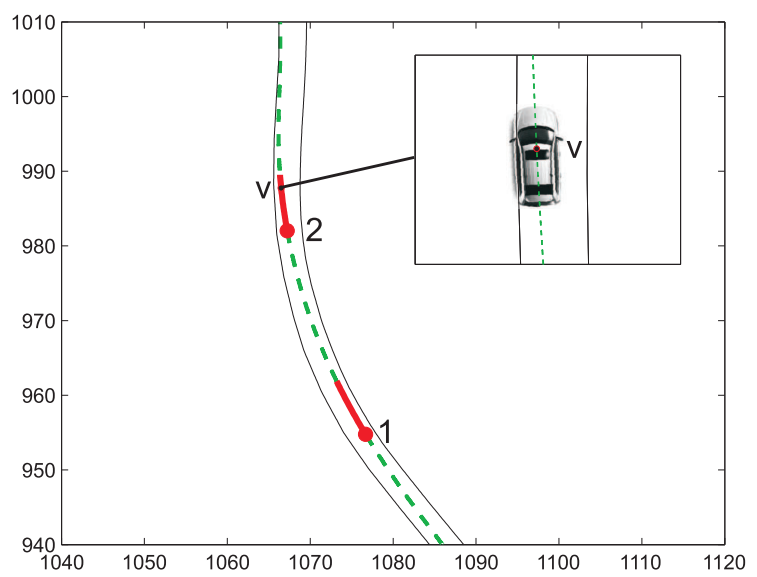

Fig. 2. Scenario where a vehicle is negotiating a curve. The dashed line denotes the vehicle trajectory. The circles denote the vehicle positions 1 and 2, respectively. The solid lines originating from the circles denote the horizon of $N$-steps.

vehicle crosses the outer lane marking, and thus violates the constraints (10), at position $V$.

In Figure 3, we show the output of Algorithm 1, i.e. the flag notSafe, for the situation in Figure 2. The results in Figure 3(a) have been computed assuming no uncertainty in the measurements of the state vector, i.e. we have set $[\mathbf{e}(k)]=[0,0]^{4}$. Denote by $k_{v}$, the time instant when the vehicle is in position $V$. Recall that, a constraint violation occurs at position $V$, hence a "nominal" flag not $S$ a f $e_{\text {nom }}$ would be raised $N-1$ steps earlier, i.e. at time $k_{a}=k_{v}-N+1$. This is illustrated with a dashed line in Figure 3. The solid line shows not Safe obtained through Algorithm 1. The vertical dash-dot lines in Figure 3(a) are placed at the time instants $k_{v}, k_{a}$ and $k_{1}$, where $k_{1}$ denotes the time instant when the vehicle is in position 1 in Figure 2. We note that the flag notSafe computed through Algorithm 1 is raised already at $k_{1}$, which is too early compared to the nominal flag.

In the results shown in Figure 3(b), on the other hand, we have accounted for uncertainty in the measurements by setting,

$$
\begin{aligned}
& {[\mathbf{e}(k)]=\left[e_{1}(k)\right] \times\left[e_{2}(k)\right] \times\left[e_{3}(k)\right] \times\left[e_{4}(k)\right],} \\
& e_{i}(k)=\left[-0.02\left|\tilde{x}_{i}(k)\right|, 0.02\left|\tilde{x}_{i}(k)\right|\right], i \in\{1,2,3,4\} .
\end{aligned}
$$

where $\tilde{x}_{i}(k)$ denotes the $i$-th component of the measured state vector $\tilde{\mathbf{x}}(k)$. We note that in this case, the flag notSafe is instead set at the time instant $k_{2}$, i.e. the time instant when the vehicle is in position 2 in Figure 2. Compared to the nominal flag notSafe $e_{\text {nom }}$ which is set at $k_{a}$, the flag notSafe computed through Algorithm 1 is here delayed 8 samples. By accounting for measurement uncertainties we managed, in this case, to remove the early intervention at $k_{1}$. The cost for the robustness against measurement errors is however in this case a small delay.

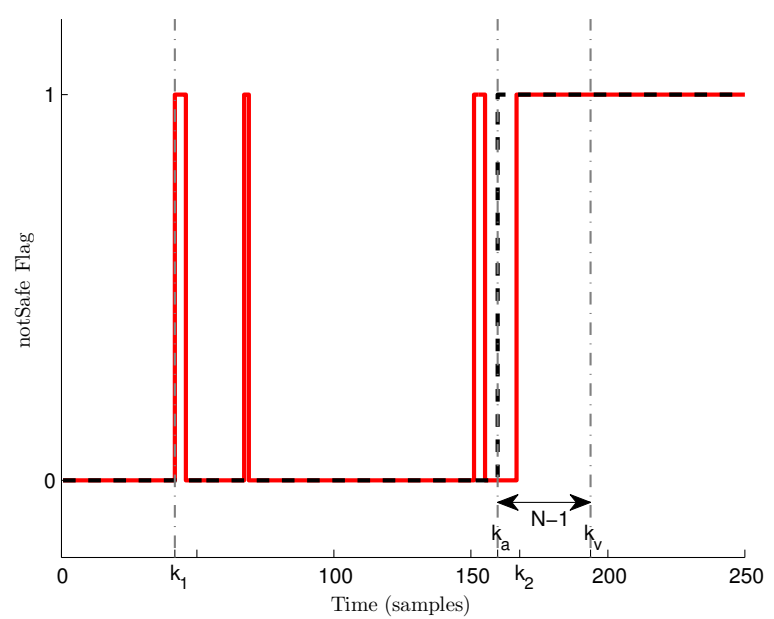

(a)

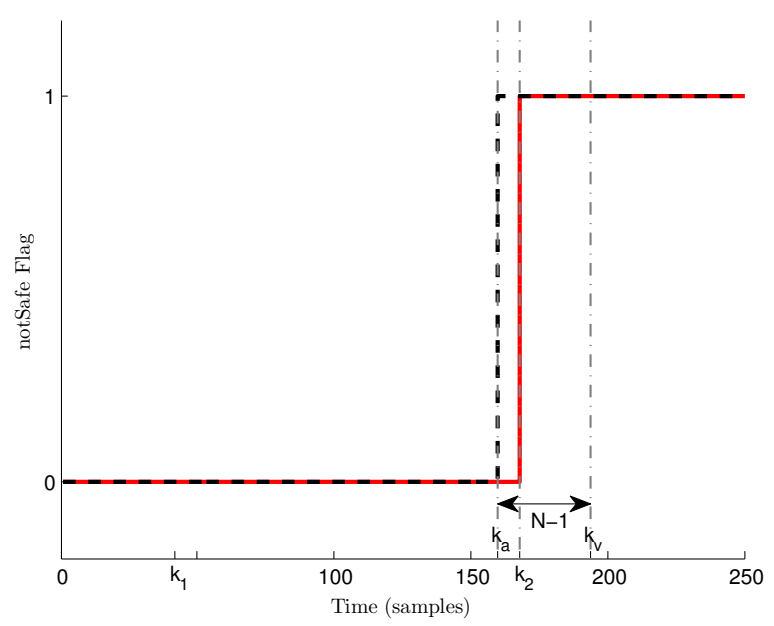

(b)

Fig. 3. Output of the proposed algorithm, the solid line denotes the flag notSafe and the dashed line denotes the flag notSafe $e_{n o m}$. In (a) no measurement uncertainty is considered, while in (b) measurements uncertainty according to (19) is considered.

In Figures 4, 5, we illustrate how the measurement uncertainty influences the threat assessment CSP. The sequences of boxes illustrate the predicted evolution of the state vector over the horizon of $N$-steps. The black boxes denote the set of possible values of the state vector at time step $k$, i.e. $\left\{\mathbf{x}(k) \in \mathbb{R}^{4} \mid \mathbf{x}(k)=\tilde{\mathbf{x}}(k)-\mathbf{e}(k), \mathbf{e}(k) \in[\mathbf{e}(k)]\right\}$, while the boxes are gradually brighter further along the horizon. $[\mathbf{e}(k)]$ has been set according to (19). The dashed line illustrates the boundary of the constraints sets (10) at time step $k+N-1$. We remark however that the illustrations in Figures 4, 5 are in 2-dimensions while the illustrated sets are of higher dimension. The sets in Figures 4, 5, are cuts at measured values of components of the state vector. In e.g. Figure 5, the black box thus denotes, 


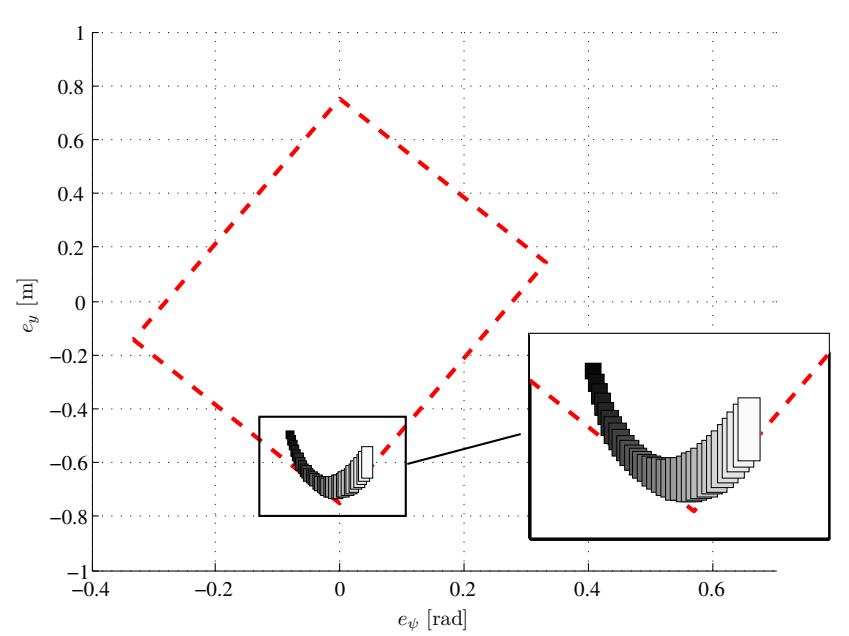

Fig. 4. The dashed line illustrates the boundary of the constraint set (10). The boxes denote the evolution of the vehicle states over the horizon of $N$-steps. The black box denotes the possible vehicle state at time $k_{1}$ while the boxes are gradually brighter further along the horizon.

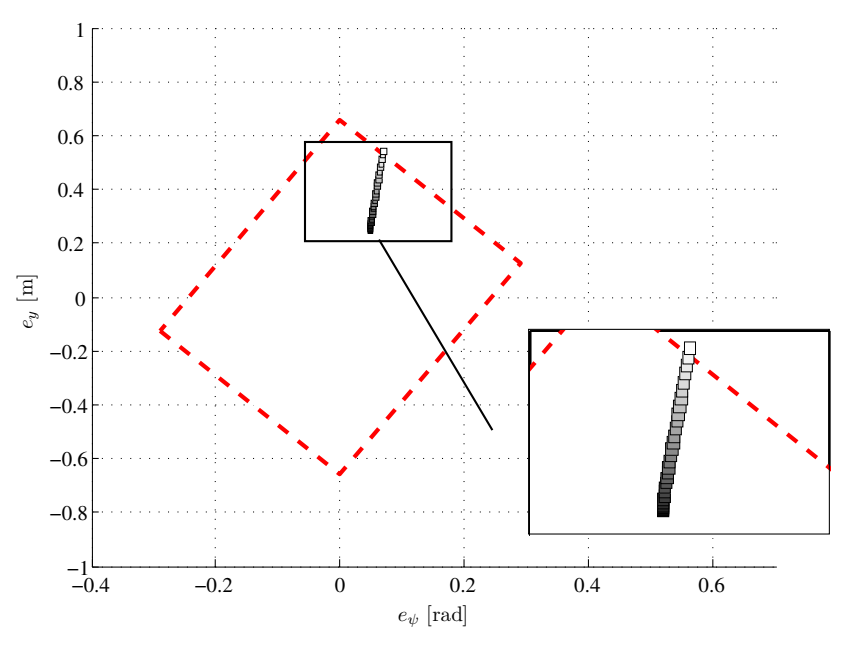

Fig. 5. The same color convention as in Figure 4 has been adopted, the evolution of the vehicle state starts at time $k_{2}$.

$$
\left.\left\{\left[\mathbf{x}\left(k_{2}\right)\right] \bigcap \mathbf{x} \in \mathbb{R}^{4}:\left[\begin{array}{llll}
1 & 0 & 0 & 0 \\
0 & 1 & 0 & 0
\end{array}\right] \mathbf{x}=\left[\begin{array}{l}
\tilde{x}_{1}\left(k_{2}\right) \\
\tilde{x}_{2}\left(k_{2}\right)
\end{array}\right]\right\}\right\} .
$$

We note that in Figure 5, the white box denoting the set of possible vehicle states at the end of the prediction horizon is disjoint with the constraint set (10), while this is not the case in Figure 4. We also observe that according to the prediction shown in Figure 5, the vehicle is expected to violate the constraint $e_{y_{f l}} \leq e_{y_{\max }}$ in particular. This is in fact confirmed in Figure 2 where we observe that the front left corner of the vehicle is the first to cross the lane marking at time step $k_{v}$.

\section{CONCLUSIONS \& FUTURE WORK}

We have presented a model based threat assessment algorithm for driver assistance systems. The threat assessment problem has been formulated as a constraint satisfaction problem, solved through interval-based methods. The experimental results shown in this paper demonstrate the proposed methods ability to predict unsafe situations, despite uncertainties in the available measurements. However, we remark that extensive testing in a larger range of conditions need to be conducted and that the adopted vehicle and driver models might not be sufficient in all operating conditions. The promising results obtained with such simple models however motivates $(i)$ further investigation with nonlinear models and constraints and (ii) accounting for uncertainties in the model parameters, in these cases the interval-based techniques are particularly beneficial.

\section{REFERENCES}

Ali, M. (2010). On Automotive Roadway Departure Prevention. Licentiate thesis, Chalmers University of Technology.

Ali, M., Olsson, C., and Sjöberg, J. (2009). Towards Predictive Yaw Stability Control. In Proc. IEEE Intelligent Vehicle Symposium.

Ali, M. and Sjöberg, J. (2009). Impact of different vehicle models on threat assessment in critical curve situations. In Proc. 21st International Conference on Enhanced Safety of Vehicles.

Benhamou, F., Goualard, F., Granvilliers, L., and Puget, J.F. (1999). Revising hull and box consistency. In Proceedings of the International Conference on Logic Programming, 230-244. Las Cruces, NM.

Cacciabue, C. (2007). Modelling Driver Behaviour in Automotive Environments. Springer.

Collavizza, H., Delobel, F., and Rueher, M. (1999). Comparing partial consistencies. Reliable Computing, 5, 213228.

Eidehall, A. (2007). Tracking and threat assessment for automotive collision avoidance. Ph.D. thesis, Linköping University.

Falcone, P., Ali, M., and Sjöberg, J. (2010). Set-Based Threat Assessment in Lane Guidance Applications. IFAC Symposium Advances in Automotive Control.

Ferguson, S.A. (2007). The effectiveness of electronic stability control in reducing real-world crashes: a literature review. Traffic injury prevention, 8(4), 329-38. doi: 10.1080/15389580701588949.

Gelso, E.R. (2009). A proposal for the diagnosis of uncertain dynamic systems based on interval models. Ph.D. thesis, Universitat de Girona, Girona (Spain).

Jaulin, L., Kieffer, M., Didrit, O., and Walter, É. (2001). Applied interval analysis: with examples in parameter and state estimation, robust control and robotics. Springer, London.

LeBlanc, D., Sayer, J., Winkler, C., Ervin, R., Bogard, S., Devonshire, J., Mefford, M., Hagan, M., Bareket, Z., Goodsell, R., and Gordon, T. (2006). Road Departure Crash Warning System Field Operational Test: Methodology and Results. Technical report, The University of Michigan Transportation Research Institute.

Moore, R.E. (1966). Interval analysis. Prentice-Hall.

Najm, W., Sen, B., Smith, J., and Campbell, B. (2003). Analysis of Light Vehicle Crashes and Pre-Crash Scenarios Based on the 2000 General Estimates System.

Rajamani, R. (2006). Vehicle Dynamics and Control. Springer. 